\title{
DOSSIÊ TEMÁTICO
}

Políticas de Formação Docente e seus Desafios na América Latina

DOI: $10.22481 /$ praxis.v14i28.3458

\section{POLÍTICAS DE FORMACIÓN DOCENTE EN ARGENTINA: UNA APROXIMACION CRITICA A LAS DEFINICIONES CURRICULARES Y DE EVALUACIÓN}

\author{
TEACHER EDUCATION POLICIES IN ARGENTINA: A CRITICAL APPROACH TO \\ THE CURRICULAR AND EVALUATION DEFINITIONS
}

\author{
POLÍTICAS DE FORMAÇÃO DE PROFFESORES NA ARGENTINA: UMA \\ ABORDAGEM CRÍTICA PARA AS DEFINIÇÕES CURRICULARES E DE AVALIAÇÃO
}

Adela Coria

Universidad Nacional de Córdoba - Argentina

\begin{abstract}
Resumen
El presente artículo pone en discusión las actuales políticas de Formación Docente (FD) en Argentina, haciendo hincapié en decisiones ligadas con la evaluación y el curriculum. En el primer apartado se propone de modo sintético un marco interpretativo que sitúa la Educación Superior en tendencias neoliberales en la región, de las que no están excluidos los Institutos Superiores de Formación Docente (ISFD) y las Universidades Públicas. En el segundo se consideran las decisiones del gobierno de la educación relativas a la trama y estrategias de las políticas actuales de FD. El tercer apartado aborda la política de evaluación de estudiantes avanzados de ISFD, sus implicancias y objeto de evaluación. Esta cuestión se entrelaza con algunas definiciones curriculares que marcan hoy la tendencia formativa oficial, que re-edita la perspectiva asumida en los años '90, y que es tratada en el cuarto apartado. Finalmente, se explicita un horizonte propositivo para la Formación Docente que recnozca a los actores principales del sistema y apunte a políticas igualitarias en educación.
\end{abstract}

Palabras clave: Educación Superior. Políticas de Formación Docente. Evaluación y Curriculum

\begin{abstract}
This article discusses the current policies of Teacher Education in Argentina, emphasizing decisions related to evaluation and curriculum. In a first section, an interpretative framework is proposed in a synthetic way that places Higher Education in neoliberal tendencies in the region, of which the Higher Institutes of Teacher Education (ISFD) and Public Universities are not excludes. In the second, the decisions of the government of education regarding the plot and strategies of the current FD policies are considered. The third section deals with ISFD's advanced student evaluation policy, its implications and object of evaluation. This issue is intertwined with some curricular definitions that today mark the official formative tendency, which re-edits the perspective assumed in the 1990s, and which is dealt with in the forth section. Finally, a proactive horizon for Teacher Education is specified that recnozes the main actors of the system and points to egalitarian policies in education.
\end{abstract}

Keywords: Higher Education.Teacher Education Policies. Assessment and Curriculum 


\section{Resumo}

Este artigo discute as políticas atuais de Formação de Professores na Argentina, enfatizando as decisões relacionadas à avaliação e ao currículo. Em uma primeira seção, propõe-se um quadro interpretativo que coloca o Ensino Superior nas tendências neoliberais na região, dos quais não são excluídos os Institutos Superiores de Formação de Professores (ISFD) e Universidades Públicas. Em segundo lugar, são consideradas as decisões do governo da educação quanto ao enredo e as estratégias das políticas atuais do FD. A terceira seção trata da política avançada de avaliação de alunos do ISFD, suas implicações e objeto de avaliação.

Esta questão está interligada com algumas definições curriculares que hoje marcam a tendência formativa oficial, que reedita a perspectiva assumida na década de 1990 e que é tratada na quarta seção. Finalmente, especifica-se um horizonte proativo para a Formação de Professores que recomeça os principais atores do sistema e aponta para políticas igualitárias na educação.

Palavras chave: Educação Superior. Políticas de Formação de Professores. Avaliação e Currículo

\section{Introducción}

Todo esfuerzo por comprender las políticas de Formación Docente (FD) en el presente $^{1}$ reclama objetivar ciertas relaciones, disputas, sentidos de las acciones y del proceso que se sostiene, en la convicción que se trata de un "cruce de racionalidades", de "variados planos en los que se despliega la acción que la asemejan a un "híbrido" (VITAR, Ana, 2006, p. 41), cuyas características serían producto de la acción combinada de decisiones de los grupos hegemónicos y las múltiples resignificaciones, rechazos, reacomodamientos y creaciones de variados sectores, organizaciones, expresiones gremiales y políticas que recrean el sentido y modos de hacer trazados.

Dichas políticas se despliegan así en distintos niveles donde se reelaboran las orientaciones más generales, produciéndose a su vez ciertos solapamientos entre la Nación y los Estados provinciales, incluso, ciertas inversiones en las apuestas -sean económicas como políticas- y también cambios sustantivos en las responsabilidades de uno y otro nivel de gestión hasta ahora dominantes.

Sobre la base del estudio de documentos oficiales y de material empírico proveniente de instituciones formadoras, en este análisis se pretende dar cuenta inicialmente y de modo muy general de una perspectiva para pensar el presente, para luego centrar la mirada en la trama -compleja, no totalmente previsible- que se viene tejiendo entre diversas y aparentemente dispersas decisiones de política de Formación Docente que se direccionan a reconfigurar de modo sustantivo el paisaje del sistema formador en no mucho tiempo.

\footnotetext{
${ }^{1}$ Las ideas principales de este trabajo fueron expuestas en el encuentro de la Red Estrado Red de Formación y Trabajo Docente concretado en Argentina, en Noviembre de 2017.
} 


\section{Educación Superior en un marco neoliberal y de confrontaciones desiguales}

Carmen García Guadilla definió ya a inicios de este siglo que «[...] la educación superior se siente acosada por fuerzas comerciales de tal naturaleza, que están logrando desestabilizar el carácter de bien público que, hasta ahora, era inherente a la educación» (GARCIA GUADILLA, 2004, citada por RODRIGUES DIAS, 2016, p. 9), como Adriana Puiggrós (2017) y Daniel Filmus y otros también lo han enfatizado en diferentes intervenciones recientemente (PUIGGROS, 2017; FILMUS et al., 2017).

Es una época donde los gobiernos teóricamente democráticos se someten a las reglas del mercado y transforman la educación en negocios rentables, abandonando su sentido social. El poder, en muchos países, sigue siendo controlado por minorías que, por medios variados, dominan la vida política. (RODRIGUES DIAS, 2016).

La reedición hegemónica del pensamiento único de quienes ejercen esa dominación y controlan así la formación de la opinión pública, va consiguiendo de manera efectiva imponer un retorno a la implementación de las teorías de la modernización. En la perspectiva del Rodrígues Días (2016), la denominada internacionalización de la Educación Superior se presenta como un proceso dirigido a facilitar la movilidad de personas, bienes y servicios e ideas, pero es de carácter desigual.

La noción de Estado se debilita o desmantela, y la interdependencia se fortalece, con polos dominantes que se encuentran en los países que controlan el conocimiento y los servicios. Ellos tienden a tener el monopolio de la creación, y los otros seguimos condenados a la imitación, sobre todo por las políticas actuales y conocidas de sujetamiento y dependencia. Un ejemplo de ello son los acuerdos económicos con empresas proveedoras de tecnología para el desarrollo de MOOCs (Massive Open Online Courses), sobre los que hay una extensa discusión crítica aunque han logrado penetrar el que se ha ido configurando como mercado de ofertas de cursos para públicos diversos y de valor discutible promovido desde algunas Universidades Públicas (Universidad Nacional de Córdoba, Facultad de Filosofía y y Humanidades, 2017).

Otros rasgos económicos y sociales son contexto de desenvolvimiento y atraviesan el mundo de la Educación Superior. El neoliberalismo, como viene ocurriendo en nuestro y otros países de la región, carga a los trabajadores con la responsabilidad de la crisis, adoptando en consecuencia una política neoliberal en relación con el mundo del trabajo que, en todas partes, tiene como resultado la precariedad y la inseguridad, política que se anuncia como promotora 
de igualdad de oportunidades pero objetivamente no puede ser pensada ni como solución justa ni democrática. Del mismo modo, la educación no puede ser vista solo como una herramienta para la formación de mano de obra barata.

La tendencia a la privatización en todos los sectores relacionados con el servicio público es un rasgo sustantivo de las políticas neoliberales y parece por el momento ser irreversible.

Asistimos asimismo a disputas sobre los modos de comprender el desarrollo. En un texto reciente, según Maristella Svampa (2015, p. 21) en América Latina observamos una nueva "gramática política" que estaría poniendo en cuestión la sustentabilidad de los actuales modelos de desarrollo y plantea otras relaciones entre sociedad, economía y naturaleza. Estaríamos atravesando en esta perspectiva una fase de globalización asimétrica, donde el modelo "extractivista neodesarrollista" pondría en cuestión la idea de sustentabilidad, o le haría adquirir un sentido débil.

En ese marco, es un deber para quienes sostenemos el sentido de una Educación Superior pública, abierta, como derecho, poder pensar sus propios problemas que contribuyan a formar para la inclusión en este mundo injustamente desigual.

Pensar, investigar y disputar, con ese atravesamiento político, son hoy significantes no excluyentes entre sí y que reclaman al menos al campo intelectual de la educación. Desde ese lugar, con ese trasfondo que pareciera por ahora ser irreversible en su tendencia, es posible mirar con alguna distancia lo que está ocurriendo con las regulaciones en el presente de la FD en el nivel superior no universitario y universitario.

\section{Trama y estrategias generales de las actuales políticas de formación docente}

El pensador costarricense Gabriel Macaya (citado por RODRIGUES DIAS, 2016, p.30, s/r) definió la estrategia discursiva de los exponentes del neoliberalismo presente como la unión de conceptos antagónicos, en frases de newspeak, como «la guerra es paz»o «la paz es guerra», «la muerte es vida», «el amor es odio», «la lealtad es traición». Utilizan esta metodología cuando tratan de cuestiones como: - Desarrollo sostenible; - Globalización con cara humana; - Carrera interdisciplinaria; - Recursos suficientes; - Previsión del futuro; Universidad privada. Y en particular utilizan palabras políticamente correctas, pero con doble sentido: - Movilidad; · Armonización; · Mejor calidad; · Cooperación.

Para nuestro caso local, eso mismo ocurre en la superficie discursiva de afirmaciones del estilo: Estamos formando docentes del futuro, o Jerarquizando y redignificando la labor 
del maestro, cuando desde el gobierno de la Ciudad de Buenos Aires se alude a las justificaciones para el cierre de los ISFD y la creación a partir de ellos de una universidad para la FD que, sabemos por memoria de los años `90, y la organización gremial nacional CTERA lo recuerda en diversos comunicados, supondrá un necesario ajuste y desestabilización laboral y salarial, la imposición de nuevos requerimientos sin garantía de contar con las las condiciones propias de la vida universitaria, en síntesis, un ensayo del que podría denominarse en jerga coloquial el "desguace" -o el desarmar por partes- del sistema formador nacional (que conserva hasta el presente los ISFD y las Universidades), en definitiva, la destrucción progresiva del sistema formador.

Podemos reconocer una trama con diversas estrategias visibles que suponen una unidad de sentido. Aunque en el discurso se siga sosteniendo la idea de la complejidad de la práctica de enseñanza, la restringe a aspectos técnicos, eliminando con sutileza las dimensiones socio-cultural y subjetiva, que confunde con la emocionalidad, en la medida de su "newspeak".

Las decisiones se adoptan sobre la base de un diagnóstico equívoco que niega o subestima los logros del pasado reciente y se asienta como parámetro de contrastación en un futuro promisorio e ilusorio, sin territorio propio y apropiado, con roles ideales -el alumno del futuro, el docente del futuro- o lo que es equivalente, sin sujetos. Se trata de un diagnóstico que solo mira el fracaso, lo que falta, aun cuando de modo políticamente correcto enuncia la normativa precedente y lo que se ha hecho hasta aquí. Para aproximar una síntesis, la política en FD ha ido tramando, en un orden tentativo, las siguientes definiciones:

1. La producción de regulaciones y legislación educativa (se encuentra en tratamiento el proyecto de LEY 1070/2017, encabezado con la denominación Políticas para el Fortalecimiento de la Formación Docente); Resoluciones del Consejo Federal de Educación (CFE); Decretos como el Sistema Nacional de Reconocimiento Académico de Educación Superior (Resolución 1870 - E/2016, 28/10/2016, publicado en el Boletín Oficial y firmado por el ex Ministro de Educación y Deportes y actual Senador Nacional Esteban Bullrich). Estas regulaciones y propuestas normativas en general, han sido fuertemente rechazadas por docentes de ISFD, sectores universitarios y gremios docentes (nacionales y de distintos estados provinciales).

2. El eje de la evaluación de estudiantes de cuarto año a través del denominado “Operativo ENSEÑAR", construido sobre la base de la definición de "capacidades", que tienen como efecto el control y una fuerte regulación del destino académico de los futuros docentes. También, políticas de evaluación en el ingreso, como en el caso del 
estado provincial de Mendoza, muy influenciado por las políticas internacionales en materia de Educación Superior.

3. Definiciones curriculares por capacidades para todos los niveles, en especial, la definición de capacidades profesionales para la formación docente inicial que aunque se niegue, tienden a autonomizarse de los saberes con los que se deberían entrelazar (podría pensarse como una reedición de políticas de los años `90, aunque profundizadas por su supuesto carácter innovador);

4. Sustitución del Programa de Formación Docente continua "Nuestra Escuela", (desarrollado por el Instituto Nacional de Formación Docente (INFoD) con múltiples ofertas de Postítulos docentes virtuales desde 2014) descentralizando en algunos casos provinciales la inversión (antes nacional), y creando ofertas aisladas de cursos, donde capacitar por capacidades, neurociencia, inteligencia emocional, cursos sostenidos con el discurso de que la capacitación situada es una prioridad, y con el correlato de dejar a cientos de colegas sin su fuente laboral;

5. Ajuste y reconfiguración del sistema formador como medidas recientes pero que se avisoraban sobre todo a partir del Operativo ENSEÑAR. Son primeros indicios el cierre de carreras en el estado provincial de Jujuy y el más reciente proyecto de creación de la Universidad de la FD en la Ciudad Autónoma de Buenos Aires (CABA), con la subsunción y cierre de 29 Institutos Superiores de Formación Docente (ISFD), que resisten ampliamente la decisión inconsulta y que desconoce la historia del sistema formador superior no universitario.

Este conjunto de medidas se ha basado en el desconocimiento de los actores principales de la FD (rectores, profesores y maestros), aunque con la presión del financiamiento nacional hayan supuesto convocatorias que fundamentan los acuerdos federales. Constituyen una formación discursiva con múltiples anudamientos, consistente, y que amerita una exploración analítica de sus trazas principales.

La aparente desarticulación o dispersión normativa y legislativa constituiría una política deliberada. Se trataría en nuestra hipótesis de una política que constriñe la formación docente y va tomando por sorpresa a los actores principales, ya que no ha sido fruto de un debate que haya puesto en el centro de la escena a los Institutos Superiores de Formación Docente y a los propios formadores y futuros docentes.

Va adquiriendo la forma de una red con múltiples ramificaciones cuya naturaleza solo reconoceremos en sus implicancias al momento en que normas y leyes sean aplicadas o 
reglamentadas específicamente, o que se vayan concretando -como ocurre- a través de un conjunto de decisiones tomadas en el Consejo Federal de Educación para dotar a las medidas de legitimidad vía el apoyo de los estados provinciales (fundamentalmente, los Ministros de Educación de los estados provinciales), obviando el debate legislativo donde son variados los actores que intervienen en un campo ciertamente heterogéneo y de disputas.

En el análisis que se propone a continuación nos centramos en dos de esas medidas, estrechamente articuladas: la evaluación de terminalidad (alumnos avanzados de ISFD) y el eje de las capacidades como perfil formativo, curriculum de la formación y del dispositivo de evaluación.

\section{La evaluación de terminalidad en la formación docente inicial}

En la superficie discursiva del proyecto de Ley 1070/2017 se ponen en evidencia las intencionalidades en materia evaluativa de los estudiantes avanzados.

El ARTíCULO 33 señala:

[...] A fin de cumplimentar lo establecido en los artículos precedentes, el Instituto Nacional de Formación Docente, implementará, en el primer bimestre de cada año, en todos los institutos del país, una evaluación integradora de conocimientos, para los estudiantes de cuarto año de las carreras de formación docente, incluyendo evaluación de contenidos y competencias profesionales para ejercer la docencia. La metodología y los ejes de la evaluación serán establecidos por el Consejo Federal de Educación, a propuesta del Instituto Nacional de Formación Docente y previa consulta con las instancias creadas por los artículos 77 (Consejo Consultivo) y 139 (Encuentros Federales) de la Ley de Educación Nacional $\mathrm{N}^{\circ} 26.206$.

En tanto, el ARTíCULO 34 va más lejos, cuando se propone que la prueba de contenidos y competencias profesionales incida en los resultados de la formación a lo largo del trayecto formativo de cuatro años, y por ende, de la acreditación final:

[...] La evaluación tendrá un objetivo de diagnóstico en sus primeras realizaciones, durante un período de tiempo que se utilizará para implementar los programas de fortalecimiento institucional previstos en esta Ley. El Consejo Federal de Educación determinará el año calendario a partir del cual la evaluación integradora pasará a ser parte del promedio general del estudiante, en condiciones en todo similar a las restantes materias del currículum. (La cursiva es nuestra). 
Sin que ese proyecto avanzara en su tratamiento legislativo por haber sido fuertemente cuestionado, el gobierno aplicó la prueba ENSEÑAR a una muestra de la población de estudiantes de ISFD, discursivamente sostenida en la idea diagnóstica. Dicho operativo de evaluación, por la tendencia marcada en el proyecto de ley, tendrá probablemente "efectos destino" de todo el proceso evaluativo del conjunto de estudiantes no solo una muestra- pudiendo llegar a condicionar la acreditación para el egreso. Como consecuencia, también tendría efectos de control adoptándose nuevos principios y jerarquías de excelencia para el egreso en un marco meritocrático de inclusiones y exclusiones (PERRENOUD, 2008), característico de las políticas neoliberales vigentes.

Es de interés reconocer en toda su dimensión el Operativo ENSEÑAR, pensar el carácter del dispositivo, los instrumentos aplicados y los efectos de rechazo y también aprobación que generó. En esta clave, lejos de estudiar herramientas aisladas, entendemos el dispositivo como red de discursos y prácticas, estrategias, técnicas e instrumentos, con efectos de sentido en los sujetos individual e institucionalmente considerados. Es decir sostenemos que es posible analizar varios componentes y a partir de ellos interrogar los supuestos en que se asienta y que producen una trama que no es reconocible a simple vista.

El primer indicio de la trama es el momento del proceso en que se decide evaluar: el último año de las carreras de formación docente genera un interrogante sobre el vínculo que esto tiene respecto de los procesos de evaluación más complejos, integrales, habituales y contextualizados que se sostienen en los ISFD, en particular, los que se observan en el espacio curricular de cierre del proceso formativo, que es definitorio en la acreditación de los estudiantes en vistas al egreso, denominada Residencia y Práctica Docente o Práctica Docente $\mathrm{IV}^{2}$

Pero de modo más crítico, nuestra hipótesis a partir del indicio del carácter muestral del operativo, es que se establecería como una estrategia exploratoria para probar confiabilidad y validez en la aplicación de instrumentos ${ }^{3}$ cuyo ulterior propósito sería muy probablemente, establecer aquella prueba de terminalidad establecida en el artículo 34 del Anteproyecto de ley en discusión ya referido, y que también ha tenido fuerte rechazo gremial.

\footnotetext{
${ }^{2}$ Dicha instancia de práctica es la última luego de un proceso en que se incluyó la Práctica Docente en el curriculum -con diversas versiones- desde el inicio del trayecto formativo. En una de las instituciones en que se aplicó la evaluación (y venimos estudiando en el caso local de la provincia de Córdoba), consultados algunos estudiantes, señalaron que aún estando en ese espacio curricular en proceso de reflexión post práctica, y elaborando el informe final, no hubo preguntas ni espacio que permitiera articular los análisis que venían realizando con las preguntas del instrumento del Operativo ENSEÑAR, la mayoría de opciones.

${ }^{3}$ En este caso de carácter estructurado, con opciones de cruce entre módulos o apartados de la prueba, de la evaluación de las capacidades o desempeños con los datos personales, con preguntas que remiten a qué se piensa de la formación recibida, por ejemplo, o las condiciones institucionales y recursos con que se cuenta.
} 
Nos preguntamos por el lugar y la imagen del docente construidos en el discurso oficial actual sobre la evaluación y cómo opera el "desconocimiento" de los saberes pedagógicos de las y los formadores de docentes en tanto actores principales que no fueron consultados en la elaboración de este dispositivo y que tenemos indicios también por los cruces que hemos mencionado, son indirectamente evaluados (ver nota 3 a pie de página).

Así, al estilo en que Viñao (2002) refiere sobre la "retórica discursiva" implícita y explícita de una reforma, se desliza un supuesto fuerte en esta evaluación ENSEÑAR: se establecería una relación de causalidad entre aquello que se enseña en los institutos formadores y lo que el instrumento construye como resultados de aprendizaje. Decimos construye pues los aprendizajes seguramente rebasan las preguntas priorizadas en la prueba sobre la enseñanza. Asimismo, la enseñanza termina siendo construida desde la visión que se juega en las opciones cerradas del instrumento, visión restrictiva y poco sensible a las múltiples realidades culturales en las que se actúa, priorizándose desempeños basados en casos.

Como operación política, este supuesto sería formativo respecto de los formadores y los futuros docentes, busca institucionalizar nuevas "identidades pedagógicas" (BERNSTEIN,1993; 1998; 2003) lo que será posible -aunque claramente hay resistenciaspor sus indudables efectos en la subjetividad (ALEMAN, 2016, p. 14).4

Forma entonces el ENSEÑAR, de modo implícito, sobre aquello que se "debe enseñar" porque será ulteriormente evaluado. Y allí emerge un interrogante fundamental sobre el dispositivo, el interrogante sobre el objeto de la evaluación, qué se evalúa, consistente con prescripciones curriculares presentes.

[...] Enseñar brindará elementos de diagnóstico del sistema de formación docente en torno al conocimiento de algunas capacidades específicas por parte de los estudiantes tomando como punto de partida los Lineamientos Curriculares Nacionales para la Formación Docente Nacional, aprobados por Resolución $\mathrm{N}^{\circ} 24$ en el año 2007 por el Consejo Federal de Educación, así como la experiencia de los institutos y las jurisdicciones en dicho campo y antecedentes en materia de evaluación.

El enfoque de enseñanza de capacidades para todos los niveles del sistema se expone en el documento "Marco Nacional de integración de los aprendizajes: hacia el desarrollo de

\footnotetext{
${ }^{4}$ Señala Jorge Aleman que "lo que le otorga su especificidad terminante al Neoliberalismo es que es el primer régimen histórico que intenta por todos los medios alcanzar la primera dependencia simbólica, afectar tanto los cuerpos como la captura por la palabra del ser vivo en su dependencia estructural (...). En este aspecto el Neoliberalismo necesita producir un "hombre nuevo" engendrado desde su propio presente, no recamado por ninguna causa o legado simbólico y precario, "líquido", fluido y volátil como la propia mercancía" (ALEMAN, 2016, p. 14).
} 
capacidades", como foco de la organización curricular de la enseñanza a los fines de favorecer procesos de aprendizaje de calidad que sostengan trayectorias educativas integrales y continuas, asentándose en el Plan Estratégico Nacional "Argentina Enseña y Aprende” (Res. 285/16). Las capacidades son -se sostiene- los recursos internos "con que" un estudiante puede lograr determinado desempeño.... Si este es el criterio para toda la educación obligatoria, queda claro hacia donde se supone se enfoca la formación de docentes para esos niveles.

En el ABC del Operativo ENSEÑAR (2017, p. 2) se definen de modo sintético las capacidades que se evalúan en estudiantes de cuarto año:

\section{$[\ldots]$ ¿Qué evalúa Enseñar?}

Enseñar combina consignas abiertas y cerradas de respuesta única y múltiple, y evalúa las áreas de Comunicación Escrita y Criterio Pedagógico.

Comunicación escrita

- Lectura (extraer información, interpretar información y reflexionar y evaluar).

- Escritura (producir textos escritos).

Criterio pedagógico

- Planificación de la enseñanza.

- Implementación de estrategias de enseñanza.

- Evaluación de los aprendizajes.

También se suministra un cuestionario complementario auto-administrado a estudiantes y directores de institutos, para conocer más en profundidad las condiciones en las que se llevan a cabo la enseñanza y el aprendizaje.

En nuestro análisis, estas capacidades remitirían en lo esencial a una dimensión instrumental del quehacer docente, con un rasgo de prescripción y detalle minimalista, con fuertes precedentes en los enfoques tecnicistas dominantes EEUU en los años `60-’70, y que fueran puestos en cuestión desde los primeros estudios etnográficos y sociológicos desde mediados de los ' 60 .

De este modo, indicadores y grillas, resultados y escalas de desempeño presentes en los sistemas de evaluación como prioridad de la política educativa nacional también refuerzan la reconfiguración del enfoque en el que se asienta la enseñanza, el lugar de las y los maestros, el vínculo pedagógico con las infancias, las familias, el territorio.

Redefiniciones curriculares: el retorno a la prioridad a las capacidades en procesos de formación y evaluación 
El Operativo ENSEÑAR descripto sería quizás la expresión más concreta de un cambio de enfoque en la formación docente inicial focalizada como decíamos en el desarrollo de "capacidades generales" que implican una visión restringida de la necesaria articulación de saberes ligados con la problematización de las heterogéneas realidades de la enseñanza en diversos contextos locales. En ese sentido, debemos observar también que las definiciones curriculares basadas en capacidades para todos los niveles, pero en especial, la definición de capacidades profesionales para la formación docente inicial tienden a autonomizarse de los saberes con los que se deberían entrelazar. ${ }^{5}$

Aunque también se haga un esfuerzo discursivo de diferenciación (Marco Capacidades, p. 6), debemos hablar del retorno del discurso de una política crucial en los '90 en la que se adoptó la noción de "Competencias", que entonces -bajo la influencia española, entre otras- dio lugar a la distinción curricular de "conceptos, procedimientos y actitudes" definidos como "saber, saber hacer, saber ser", visión que en el contexto socio-político neoliberal de los `90s se desplegó como una política vinculada con la lógica de mercado dominante en la vida social y en el discurso globalizador, y olvidando la complejidad que pregonaba, cayó en reduccionismos poco sensibles a las problemáticas pedagógicas.

Que hoy se establezcan las mismas capacidades para todos los niveles del sistema y para todo contenido de enseñanza, sería el indicio privilegiado de esa autonomización de los saberes con los que se vinculan -aún cuando se los nombre-, de las lógicas de producción de esos saberes, y radicalmente, se descontextualizan desde un punto de vista histórico-social y cultural.

Priman los "desempeños", un claro vaciamiento que apunta a la formación de individuos donde importa la eficacia de las actuaciones y el emprendedurismo.

El retorno al discurso de las capacidades, puesto en evidencia también en el Plan Maestro, y en los que se definen para la Formación Docente inicial como "recursos internos para lograr desempeños", se orienta a organizar el currículum, la enseñanza y la evaluación los tres sistemas de mensaje a través de los cuales Basil Bernstein (2003) analiza el tallado en la subjetividad y la transmisión implícita de ideología, a través de los códigos prevalentes- en torno a un conjunto de capacidades consideradas centrales y para la formación docente, como

\footnotetext{
${ }^{5}$ En el proyecto de ley citado, en su ARTÍCULO $7^{\circ}$, se señala: El Consejo Federal de Educación, a propuesta del Instituto Nacional de Formación Docente, aprobará criterios y parámetros referidos al perfil profesional docente, así como sobre conocimientos y competencias esperables en los egresados de la formación docente, que constituirán el marco de referencia para la estructuración de ofertas formativas o planes de estudio que accedan al reconocimiento de validez nacional por parte del Ministerio de Educación y Deportes.
} 
transversales: Resolución de problemas, Pensamiento crítico, Aprender a Aprender, Trabajo con otros, Comunicación, y Compromiso y responsabilidad.

Así como habría una perspectiva reductiva y eficientista sobre el quehacer docente, también se interpreta una reducción de los sujetos a alguna versión de niños y adolescentes en su exclusiva dimensión cognoscente de adquisición de alguna habilidad o estrategia cognitiva y emocional y ya no sujetos de deseo, sociales y culturales, con historia. En últimainstancia, estaríamos de cara a la ausencia de pedagogía, y a la enseñanza solo basada en formas de entender el aprendizaje, lo que habrá de evaluarse.

En el ABC del Operativo ENSEÑAR, ya en relación con los docentes en ejercicio en los párrafos que se dedican a la planificación, se minimiza el trabajo intelectual del docente, dejándolo en el lugar de diseñador de un proceso: “[...] El proceso, entonces, va desde la selección de unas capacidades a enfocar, hasta el diseño de procedimientos de evaluación para conocer si el alumno ha desarrollado dichas capacidades" (ABC, p. 11).

Así como el discurso de las competencias reenvía claramente a los'90, el de las "capacidades" como organizador curricular, auguradas como novedad para la Argentina 2030, no es más que una repetición y retroceso en la imaginación pedagógico-didáctica: a nivel de la agencia de construcción del discurso pedagógico oficial nacional se sostuvo literalmente entre los años 2001-2003 como política curricular tanto para los niveles primario y secundario como para la formación docente. Luego hacia 2010 se articuló como el objetivo pedagógico principal desde organismos no gubernamentales, fundaciones $\mathrm{u}$ otros organismos privados con influencia en materia educativa, y con proyección en algunas provincias (Formosa, Chaco, Jujuy, Misiones, Córdoba), a través de producciones en las que intervinieron agentes que hoy ocupan posiciones destacadas en el Ministerio de Educación Nacional.

En el Marco Referencial de Capacidades Profesionales se sostiene:

[...] La formación inicial debe garantizar el logro de las capacidades profesionales en un nivel básico que será ampliado y profundizado posteriormente, a lo largo de la extensa trayectoria laboral prevista para los egresados. Un docente experto tendrá un mayor bagaje de este tipo de capacidades, podrá actuar en una variedad mayor de situaciones e intervenir en ellas con mayores niveles de complejidad. Pero la formación inicial debe hacerse responsable de identificar un conjunto básico, indispensable para comenzar un camino profesional que deberá ser apoyado luego por las instituciones, los equipos directivos, los colegas más experimentados y los dispositivos de acompañamiento desarrollados a nivel nacional, jurisdiccional e institucional. (2017, p. 6-7)

Estas capacidades profesionales se enuncian en los siguientes términos: 
[...] 1.Dominar los saberes a enseñar;

2. Actuar de acuerdo con las características y necesidades de los sujetos y de sus procesos de aprendizaje;

3. Dirigir la enseñanza y gestionar la clase;

4. Intervenir en la dinámica grupal y organizar el trabajo escolar;

5. Intervenir en el escenario institucional y comunitario;

6. Comprometerse con el propio proceso formativo.

Por los significantes en uso - dirigir y gestionar -, es en la tercera capacidad donde más se pone en evidencia el enfoque de una nueva tecnología aplicada a la enseñanza en juego, pues además, se define como la dimensión central de la profesión docente.

En su alcance, se define que refiere a las acciones de guía, ayuda y a la conducción de las tareas de aprendizaje, en los escenarios específicos, decidiendo sobre estrategias, recursos, tiempos, espacios y agrupamientos. "Esta dimensión -se argumenta- involucra el proceso de planificación, el desarrollo efectivo de las clases y la evaluación de los aprendizajes". ${ }^{6}$

Es sintomático que las complejas realidades sociales, culturales, territoriales e identitarias queden elididas del discurso, y se subsuman a aquellos ya muy viejos significantes de la enseñanza como guía, ayuda -aparentemente débiles y secundarios- conjuntamente con la denominada conducción del aprendizaje -esos vínculos de "new-speak", para volver al inicio de este análisis- que reúnen trazando idearios formativos difícilmente cuestionables o políticamente correctos, que interpelan por la crítica implícita al pasado en que se sostienen y que desdibujan la enseñanza en su alcance político, cultural, subjetivo y moral.

\section{Para un cierre: seguir habilitando futuro}

Aún con tensiones y contradicciones, en Argentina entre 2004 y 2015 hubo un tiempo altamente productivo que rechazó la centralidad del discurso de la reforma curricular como garante casi exclusivo de una pretendida transformación educativa-discurso que olvidó sujetos y procesos escolares-, y produjo algunos aportes desde las políticas de enseñanza que hoy se reconocen en su vigencia en muchas aulas y escuelas del país.

En los últimos años, como formadores, trabajamos en la recuperación de la pedagogía y sus referencias buscando habilitar relaciones en los procesos formativos con diferentes sistemas simbólicos; en pensar a nuestros estudiantes futuros docentes como

\footnotetext{
${ }^{6}$ En el Anexo de dicho documento se especifican las capacidades profesionales que según se sostiene, habrán de orientar la definición de capacidades para trazar los perfiles docentes en los distintos estados provinciales. (Anexo Capacidades Profesionales para la Formación Docente Inicial, 2017, p. 9-10).
} 
sujetos de apropiación y productores de interpretaciones, valoraciones, modos de actuación sobre el mundo que los rodea; atendiendo en particular la dimensión del deseo y de las representaciones sociales de las que son portadores en tanto sujetos. Trabajamos asentados en el reconocimiento de la autoridad intelectual de los futuros docentes y formadores para apropiarse de saberes desde la perspectiva de los desafíos culturales de la contemporaneidad (BIRGIN, 2006), lejanos a la banalidad a la que ha llegado el reiterado enunciado del "trabajo en equipo" propio del discurso empresarial, o a las habilitaciones cognitivas de los sistemas neuronales, en las versiones hoy también banalizadas de las neurociencias.

Veníamos haciendo un esfuerzo desde el punto de vista didáctico de unir lo que se había separado en la reforma curricular de los `90 (como señalamos, conceptos, procedimientos y actitudes) y que sesgó el modo de pensar de los docentes, plasmándose en las planificaciones de aula. Veníamos procurando dar cuenta de un enfoque relacional sobre la enseñanza de cada campo disciplinar y por ende, de un estilo de trabajo intelectual donde recuperar los saberes sustantivos, los modos de proceder al investigarlos y validarlos y los valores que están en juego en todo acto de conocimiento. (BOURDIEU, GROS, 1989).

Veníamos procurando pensar el carácter de invención y acontecimiento que supone el enseñar, y en el valor del enseñar.

Hoy, vemos con preocupación que aquellos procesos mentales largamente cuestionados como único sostén curricular, retornaron con la legitimidad que parecen darle el desiderátum de lo "verdaderamente científico", volviendo a esquematismos en las planificaciones docentes y diseños curriculares que olvidan los saberes objetos de enseñanza por la obsesión de las capacidades, invisibilizan las condiciones escolares en que se despliega la enseñanza y los procesos socio-culturales y cognitivos de niños y adolescentes, y se deslizan a convertir a los docentes en meros aplicadores.

Son numerosos los emergentes que dan cuenta de una reconfiguración del enfoque en el que se asienta la formación docente, la enseñanza, el lugar de las y los maestros, el vínculo pedagógico con el contexto, emergentes que muestran que se ha producido un giro profundo en las propuestas formativas del Ministerio de Educación y Deportes de la Nación, avanzando en la "instrumentalización" del pensamiento pedagógico, lo que supone al mismo tiempo la eliminación de la pedagogía y la enseñanza como prácticas políticas (lo que está en juego no es el desarrollo de unas capacidades, sino compartir saberes valiosos y la inscripción subjetiva de niños, jóvenes y también adultos, en una matriz simbólica). 
Hoy como formadores avanzaremos construyendo alternativas ante esas efímeras y estentóreas fórmulas de marketing para el campo editorial y las escuelas que subestiman la estatura intelectual de los docentes en ejercicio y formación.

Hoy la memoria pedagógica reciente disputa con los sesgos neoliberales, meritocráticos y conservadores del actual discurso educativo que llevan a profundizar desigualdades injustas en la educación y a la hipercentralización del ejercicio del poder a través de la evaluación sobre los estados provinciales y del control social de sus instituciones, maestros y profesores, de sus alumnos y alumnas y sus familias.

Es desafío para un importante colectivo de educadores pensar cómo ir habilitando cada vez más actos políticos ante este alud que arrasa en muchos sentidos prácticas formativas, curriculares e instituciones en el marco de procesos represivos y de transformaciones traumáticos bajo un velo de redignificación.

La tarea es y será ardua. Pero confiamos en la búsqueda de una formación, educación y escuela justas en las que el disfrute con el conocimiento, las artes, las ciencias, la literatura, la historia y la búsqueda de nexos renovados entre saberes no tengan como meta lo que podrá ser evaluado, sino expandir experiencias pedagógicas cuya riqueza y amplitud de miras proyecten un porvenir igualitario, sin exclusiones.

Volver a la pedagogía y la enseñanza en clave política, una y otra vez, defender nuestros logros históricos en la formación docente por sobre la hegemonía del evaluar del aprender y los cierres con promesas demagógicas y confusas, es tarea, es reto, es ilusión, es hoy deber para el conjunto de la docencia y en especial para las investigaciones que se sostengan desde el campo intelectual de la educación (DIAZ, 1992).

Fuera de todo esencialismo, es nuestro horizonte revisar críticamente las apuestas que orientaron nuestro accionar pero asentarnos en las trayectorias colectivas y sostener una batalla simbólica contra las "identidades pedagógicas" que esta reforma pretende institucionalizar porque tenemos historia y conciencia de sus poderosos efectos en nuestras instituciones y la subjetividad.

La resistencia de las instituciones y las producciones de los formadores, maestros y profesores para enseñar, en todo el país, dan cuenta de los esfuerzos por sostener, en situaciones muchas veces adversas, la apuesta político-pedagógica por una enseñanza emancipadora, como el signo de despegue de la antiguonovísima tecnología de las capacidades atravesando todo el sistema.

Tenemos como trasfondo el imaginario de la "escuela pública" como un lugar de referencia social, ámbito de transmisión de la cultura, espacio de lo público y lo común, lugar 
de sobrevivencia, inventiva y construcción identitaria que garantizó que los sujetos continuaran (en los contextos más críticos) creyendo en la educación.

Confiamos en las y los maestros y profesores, presentes y futuros, como "productores" de saberes culturales y pedagógicos, capaces de articular su formación y su experiencia, capaces de valorar y proyectar alternativas de enseñanza en sus aulas y escuelas. Y un histórico reconocimiento que habilita: la "autorización" de las y los maestros "citados a inventar”. (MEIRIEU, 2001, pp. 104-106;CORIA, 2014; DE CERTEAU, 1996).

Es nuestro desafío hoy reconducir el discurso y las prácticas, disputando nuevamente, como en los `90s, esa idea neoliberal pero también neoconservadora de fin de la escuela, fin de la enseñanza, fin de la pedagogía, sus preguntas, sus atravesamientos, sus siempre acuciantes dilemas...

\section{Referencias}

ALEMAN, Jorge. Horizontes neoliberales en la subjetividad. Buenos Aires: Grama, 2016.

BERNSTEIN, Basil. La estructura del discurso pedagógico. Madrid: Morata, 1993.

BERNSTEIN, Basil. Pedagogía, control simbólico e identidad. Madrid: Morata, 1998.

BERNSTEIN, Basil. Clases, códigos y Control. Hacia una teoría de las transmisiones educativas. Madrid: Akal Universitaria, 2003.

BIRGIN, Alejandra. "Pensar la formación docente en nuestro tiempo". En TERIGI, Flavia. Diez miradas sobre la escuela primaria. Buenos Aires: Siglo XXI, 2006.

BOURDIEU, Pierre Y GROS, François. Principios para una reflexión sobre los contenidos de enseñanza. Traducción de Margarita Krap, 1989. Disponible en https://es.scribd.com/document/143668864/Principios-para-una-reflexion-sobre-loscontenidos-de-la-ensenanza.

CORIA, Adela. Entre curriculum y enseñanza. Aristas de un proceso político-pedagógico en la construcción de la política curricular de la enseñanza en Argentina (2004-2007). En Estela M. Miranda y Newton Paciulli Bryan. Formación de profesores, curriculum, sujetos y prácticas educativas en Argentina y Brasil. Córdoba: Facultad de Filosofía y Humanidades - UNC. E-Book: 2014. www.ffyh.unc.edu.ar/editorial/

CORIA, Adela. La clase imaginada: “citados a inventar”. Módulo Prácticas de Enseñanza con TIC. Colaboradoras: Paula Basel, Mariana Palmero. Especialización docente de nivel superior en Educación Primaria y TIC. Buenos Aires: Ministerio de Educación de la Nación, 2014.

DE CERTEAU, Michel. La invención de lo cotidiano. Artes de hacer. México: UIA, 1996. 
DIAZ, Mario. “Aproximaciones al campo intelectual de la educación.” En: Larrosa, Jorge (ed.) Poder, Escuela y Subjetividad. Madrid, Ed. La Piqueta, 1992.

EDELSTEIN, Gloria y CORIA, Adela. Imágenes e imaginación. Iniciación a la docencia. Kapelusz. Buenos Aires, 1995.

FILMUS, Daniel (Comp.), Educar para el mercado. Escuela, Universidad y Ciencia en tiempos de neoliberalismo. Buenos Aires: Octubre, 2017. Ver entrevista a Daniel Filmus, Graciela Morgade y Sandra Carli en https://www.pagina12.com.ar/54980-buscan-enfocar-laeducacion-en-el-mercado. Consultado el 20 de diciembre de 2017.

GARCÍA GUADILLA (Edit.), C. (2004). El Difícil equilibrio. La educación superior como bien público y comercio de servicios: implicaciones del AGCS (GATS). Universidad de Lima y Columbus. En: MARCO ANTONIO RODRIGUES DIAS. Enseñanza superior como bien público. Perspectivas para el centenario de la declaración de Córdoba. Montevideo: AUGM, 2016.

MEIRIEU, Philippe. La opción de educar. Etica y Pedagogía. Barcelona: Octaedro, 2001. PERRENOUD, Philippe. La evaluación de los alumnos. De la producción de la excelencia a la regulación de los aprendizajes. Entre dos lógicas. Buenos Aires: Colihue, 2008.

MEIRIEU, Philippe. El trabajo sobre el habitus en la formación de maestros. Análisis de las prácticas y toma de conciencia. En Paquay, L., Altet, M.; Charlier, E.; Perrenoud, P. (Coords.). La formación profesional del maestro. Estrategias y competencias. México: FCE, 2005.

PUIGGROS, Adriana. Adiós Sarmiento, Educación pública, Iglesia y Mercado. Buenos Aires: Colihue, 2017.

SVAMPA, Maristella. El desarrollo en disputa. Actores, conflictos y modelos de desarrollo en la Argentina contemporánea. Buenos Aires: UNGS, 2015.

RODRIGUES DIAS, Marco Antonio. Enseñanza superior como bien público. Perspectivas para el centenario de la declaración de Córdoba. Montevideo: AUGM, 2016.

VIÑAO, Antonio. Sistemas educativos, culturas escolares y reformas. Madrid: Morata, 2002.

VITAR, Ana. "Las políticas y lo(s) político(s). En Vitar, Ana (Coord.) et al. Políticas de educación. Razones de una pasión. Buenos Aires: Miño y Dávila-OEI, 2006.

\section{Documentos Consultados}

Argentina Enseña y Aprende. Plan Nacional de Acción 2016-2021. Buenos Aires: Ministerio de Educación y Deportes de la Nación, 2016. Disponible en http://educacion.gob.ar/data_storage/file/documents/argentina-ensena-y-aprende59a0468d66579.pdf. Consultado el 15 de diciembre de 2016. Anexo de la RES del CFE Nro. 285.

Enseñar. Evaluación diagnóstica de estudiantes del último año en Argentina. Documento de trabajo. Buenos Aires: Secretaría de Evaluación Educativa. Ministerio de Educación de la 
Nación, 2017. Disponible en

https://www.argentina.gob.ar/sites/default/files/documento marco ensenar 1.pdf Consultado el 30 de Octubre de 2017.

Enseñar. El ABC del Enseñar 2017. Buenos Aires: Secretaría de Evaluación Educativa. Ministerio de Educación de la Nación, 2017. Disponible en https://www.argentina.gob.ar/sites/default/files/preguntas-ensenar2017.pdf. Consultado el 30 de Octubre de 2017.

Hacia un marco referencial de Capacidades Profesionales de la Formación Docente Inicial. Buenos Aires: INFoD, 2017. Disponible en https://red.infd.edu.ar/articulos/se-presento-elmarco-referencial-de-capacidades-profesionales-de-la-formacion-inicial/ Consultado el 3 de Noviembre de 2017.

Marco Nacional de Integración de los Aprendizajes: hacia el desarrollo de capacidades. Ministerio de Educación y Deportes, 2016. Disponible en http://www.mendoza.edu.ar/wpcontent/uploads/2017/03/Capacidades.pdf. Consultado el 15 de mayo de 2017.

Proyecto de LEY 1070/2017 Políticas para el fortalecimiento de la formación docente. Senado de la Nación. Secretaría Parlamentaria. Dirección de Publicaciones. Disponible en http://www.senado.gov.ar/parlamentario/comisiones/verExp/1070.17/S/PL. Consultado el 31 de mayo de 2017.

Plan Educativo Maestro. Buenos Aires. Ministerio de Educación y Deportes de la Nación, 2017. Disponible en https://dialogo.compromisoporlaeducacion.edu.ar/...planmaestro/ProyectodeLeyPlan... Consultado el 30 de marzo de 2017.

Universidad Nacional de Córdoba, Facultad de Filosofía y Humanidades. Consideraciones críticas sobre el acuerdo UNC edX-inc desde la perspectiva de democratización del conocimiento público. Documento elevado al Honorable Consejo Superior, 2017. Mimeo.

\section{SOBRE A AUTORA:}

\section{Adela Coria}

Doutora en Ciencias con Especialidad en Investigaciones Educativas, DIE-CINVESTAV, México. Profesora Titular y Directora del Doctorado en Ciencias de la Educación de la Facultad de Filosofía y Humanidades, Universidad Nacional de Córdoba, Argentina. Integrante de la Red "Diversificación y diferenciación institucional de la formación de los docentes en el Nivel Superior del MERCOSUR. Hacia una perspectiva comparada para la construcción regional". NEIES-MERCOSUR EDUCATIVO-SPU. E-mail: coriaadela@hotmail.com 